\title{
REVIEW
}

\section{Risk reduction through skeletal scintigraphy as a screening tool in suspected scaphoid fracture: a literature review}

\author{
D Chakravarty, J Sloan, J Brenchley
}

Emerg Med J 2002;19:507-509

Fractures of the scaphoid are the most common carpal injuries. Suspected scaphoid injuries make up a large part of the work of an accident and emergency department, and unfortunately make up a significant percentage of litigation claims. Initial radiographs cannot always detect fractures while undisplaced and subsequently untreated fractures may give rise to premature carpal collapse and degenerative arthritis with alterations in the kinematics of the wrist. The use of scintigraphy in the diagnosis of fractures of the carpal scaphoid has been used routinely as a valuable supplementary examination. It has been shown to be more sensitive than routine radiography and it has been suggested that the results of early bone scanning should guide the treatment of patients with suspected scaphoid fractures. This literature review aims to look at the various studies performed regarding the use of bone scanning in suspected scaphoid fractures and an attempt to formulate a management protocol is made.
See end of article for authors' affiliations

Correspondence to: Dr D Chakravarty, 42 Abingdon House, Adrian Way, Long Road, Cambridge CB2 2SA, UK; debasischakravarty@ hotmail.com

\section{PATHOPHYSIOLOGY}

The scaphoid is the carpal bone most commonly fractured. The unreliability of radiological examination in excluding or confirming a suspected scaphoid fracture after carpal trauma is a well recognised diagnostic problem.

Complications with scaphoid fractures arise because of its vulnerable blood supply, irregular shape, peculiar oblique alignment across the plane of motion of the mid-carpal joint, and complex function as a link between the proximal and distal carpal rows. Non-union of the scaphoid can result in major changes in the kinematics of the wrist.

The proximal pole of the carpal scaphoid has been compared with the heads of the femur and talus, because it is covered completely with hyaline cartilage and has a nutritional source that may be interrupted by fracture. Hence it is important that these fractures are diagnosed early and specific treatment started so as to prevent premature carpal collapse and degenerative arthritis. Even when promptly and adequately treated, there is a risk of non-union of between $5 \%$ and $12 \%{ }^{1}$

\section{CLINICAL IMPLICATIONS}

In accident and emergency (A\&E) practice, assessment of the painful wrist after trauma is important for a number of reasons. Firstly, such injuries are of high volume, and therefore changes to the cost of individual treatment will have a major effect on overall treatment costs. Secondly, the clinical risk associated with misdiagnosis is significant. In a seven year period at our own hospital there were 21 attempts at litigation, four of which involved the scaphoid. Large settlements have been avoided, but the potential for significant payouts exists on the basis of loss of earnings in a young adult. Courts may agree sums of between $£ 50000$ to $£ 100000$ in such circumstances.

\section{DIAGNOSIS}

Papers reporting trials of bone scan in the initial diagnosis of scaphoid fracture were examined. All these studied only small numbers of patients. The absence of a recognised gold standard for the diagnosis makes papers difficult to compare. Scaphoid fractures can only be excluded definitively at open surgery or postmortem examination. Most studies use a combination of radiographs, both at initial presentation and at follow up 10 to 14 days later, combined with clinical examination to exclude the diagnosis. In these studies the prevalence of scaphoid fracture in those with initially normal radiographs ranges from $10 \%$ to $36 \%$.

\section{INITIAL RADIOGRAPHY}

An adequate initial radiographic examination of the wrist with suspected scaphoid tenderness includes neutral, ulnar deviation, posterioranterior, and lateral radiographs, as well as oblique radiographs made with the wrist in pronation. ${ }^{2}$ Fractures of the scaphoid cannot always be seen on the initial radiographs. ${ }^{3}$ In one study wrist fractures were missed on $35 \%$ of initial radiographs, half of these were scaphoid fractures. ${ }^{4}$

\section{THE ROLE OF SCINTIGRAPHY}

The use of scintigraphy in the diagnosis of fractures of the carpal scaphoid has received increased attention in recent years. Bone seeking radiopharmaceuticals are selectively taken up and concentrated at sites of fracture, producing a characteristic "hot spot". This phenomenon arises from the affinity of such agents for areas of increased osteoblastic activity or hyperaemia and is observed in bone trauma of varying severity. ${ }^{3}$ Increased isotope accumulation is also associated with neoplasm, infection, arthritis, increased 
blood flow associated with sympathetic denervation and the healing phase of aseptic necrosis. Haematomas, synovitis, and surgical incisions can lead to misleading accumulations. Most fractures will be visible on bone scanning about three to five days after trauma, but in elderly or debilitated patients this may be delayed to seven to ten days. ${ }^{5}$

Jorgensen $e t a l^{4}$ studied 50 patients with clinical suspicion of a scaphoid fracture using both radiography and isotope scanning. They had no false negative results on bone scanning.

Ganel $e t a l^{6}$ studied 49 patients with suspected fracture of the scaphoid. Radiographs were initially non-diagnostic in 31 wrists and normal in seven. Six of these had positive bone scans, and in five a fracture was later demonstrated radiographically.

Rolfe $e t \mathrm{al}^{7}$ explored the role of isotope bone imaging in the identification of carpal bone injury. In the study of 99 patients sensitivity of bone scanning was $100 \%$ and specificity $91 \%$. They conclude that a fracture can be confidently excluded in patients who show no abnormality on scintigraphy. They noted that imaging patients in the first 48 hours after injury might be misleading because of increased uptake from traumatic synovitis.

Stordahl et $_{\text {al }} \mathrm{l}^{8}$ from their study of 30 patients concluded that a fracture site may become positive on bone scan shortly after a traumatic event, and if the scan is normal at 72 hours after the event, a fracture may be excluded. Their findings confirmed that a negative bone scan excluded a fracture.

Wilson et al ${ }^{9}$ studied the practicality of using scintigraphy routinely in the diagnosis of radiograph negative suspected scaphoid fracture. The study confirmed the accuracy of a negative bone scan as a screening tool and that in the presence of a negative bone scan, the clinician may confidently mobilise the patient despite prolonged snuffbox tenderness. Hence the study concluded that bone scanning is a practical investigation for these patients.

Brismar $^{10}$ published the results of a retrospective study of skeletal scintigraphy performed in 187 patients with clinical suspicion of a scaphoid fracture but with normal radiological findings. The study confirmed that skeletal scintigraphy is of value to demonstrate scaphoid fractures before they are visible radiographically. It was also suggested that a viable policy from both the patient's and an economic point of view would be to schedule the patient for a skeletal scintigraphy at the next clinic visit two weeks after the trauma. The patient should then, however, be re-examined without cast directly before the scintigraphy and the scintigraphy cancelled if either the clinical findings had normalised or the skeletal films had become positive.

Young et $a l^{11}$ published a study performed to identify whether there is a correlation between isotope bone scanning, standard radiographs, and the clinical examination of patients presenting with suspected fractures of the scaphoid. They concluded that a normal bone scan excluded a fracture and that there was a stronger correlation between the clinical findings and bone scanning than with standard radiographs. Bone scanning also gave an earlier diagnosis than the radiograph (that is, 10 days compared with three weeks).

Tiel-Van Buul et al ${ }^{12}$ again reviewed the clinical consequences, after one year, of managing suspected scaphoid fractures according to the bone scan results. On reviewing patients at 12 months after injury, none of those with a negative bone scan result had any radiological evidence of old healed fractures. They recommended that those with negative initial radiographs should have a bone scan at 72 hours, and then be managed according to the result of that scan.

Vrettos $\mathrm{et}_{\mathrm{al}} \mathrm{l}^{13}$ conducted a prospective study on 50 patients to evaluate the role of radionuclide bone scanning in patients with suspected scaphoid trauma with negative initial radiographs. The overall positive predictive value of scintigraphy was $93 \%$. All patients with a negative scan were clinically and radiologically negative at two weeks (negative predictive value

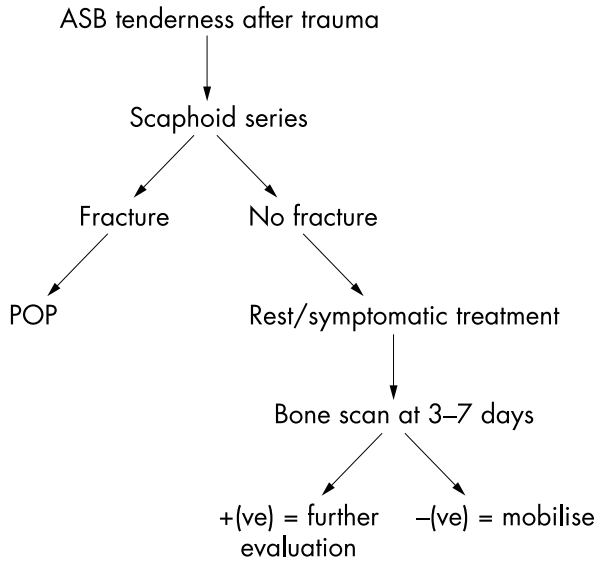

Figure 1 A suggested approach.

$100 \%$ ). They concluded that bone scintigraphy can be used reliably in radiologically negative scaphoid injury to exclude the need for further follow up, but those with positive scans still require clinical examination and radiography at two weeks.

Murphy et $a l^{14}$ studied 99 patients with anatomical snuffbox tenderness and a mechanism of injury compatible with scaphoid trauma but normal initial radiographs. All patients underwent a bone scan on day 4, the results of which were compared with the "gold standard" of clinical assessment, repeat radiography, and in symptomatic patients a further bone scan. Imaging on day 4 was 100\% sensitive for scaphoid injury with no false negative results. Specificity was $92 \%$ for scaphoid fracture, but a large number of other fractures were found.

All these studies show that in patients with a normal bone scan result, no scaphoid fracture could be demonstrated radiologically. There is less agreement on how to classify the patient with a hotspot on the scan localised to the scaphoid who consistently has normal radiographs. Bone scanning will also indicate other injuries within the carpus.

\section{ROLE OF MAGNETIC RESONANCE IMAGING (MRI)}

Several studies have examined the use of MRI in the diagnosis of suspected scaphoid injury. Tiel-van Buul et al ${ }^{15}$ compared MRI to bone scintigraphy in 19 patients with suspected scaphoid fracture with normal initial radiographs. One patient with a confirmed scaphoid fracture had a negative MRI and positive bone scan, and one patient had a lunate fracture at operation, which was correctly diagnosed only on the bone scan. Kukla et al $^{16}$ performed MR scanning one to six days after injury. They had no false negative results with MRI and diagnosed other carpal bony and ligamentous injuries with greater frequency than standard radiographs. They suggested that in their hospital an initial MR scan is cost effective compared with the standard management protocol of prolonged immobilisation and repeated outpatient assessment ( $£ 335$ compared with £320). Lohman et al ${ }^{17}$ compared MRI and radiography in 67 patients with wrist trauma. MRI diagnosed $65 \%$ more fractures than plain radiography ( 37 to 24 ) of which three involved the scaphoid. Four fractures of the triquetral bone were detected on radiography but not on MRI. There were no false negative scaphoid fractures on MRI. Hunter et $a l^{18}$ studied 36 patients with suspected scaphoid injury and normal initial radiography. MR scanning diagnosed 22 occult fractures, 13 involving the scaphoid. Eleven of these had follow up radiography and evidence of healing was seen in 10 (91\%). 


\section{CHOOSING A STRATEGY}

How does this help us to choose an effective and low risk strategy for the diagnostic management of suspected scaphoid fracture? We know that initial radiographs have a sensitivity of $59 \%,{ }^{19}$ and a large number of patients are overtreated as a precaution. Bone scintigraphy in all these studies has been shown to be $100 \%$ sensitive and would meet the requirements of a screening test. Although use of bone scintigraphy in the diagnosis of scaphoid fractures leads to an increase in diagnostic costs, therapeutic and litigation costs would inevitably be reduced. The most efficient approach seems to be a combination of first day scaphoid radiography and bone scintigraphy performed at least 72 hours after injury. Local availability of scintigraphy or MRI may influence choice of a diagnostic strategy.

\section{CONCLUSION}

The diagnostic methods available in suspected scaphoid fracture will continue to generate discussion and research. The aim of this review has been to highlight that it is possible to arrive at a diagnosis early, while probably reducing costs. To limit the number of patients immobilised unnecessarily with the consequent socioeconomic costs we need a strategy that is reliable and cost effective. Diagnostic and therapeutic costs, period of immobilisation, and complication rates can all be calculated. The true significance of a hotspot on the bone scan combined with a negative radiograph has to be established in a management study to avoid over treatment. Both scintigraphy and MRI may streamline management of the patient with suspected scaphoid fracture, but there is the need for a large, well designed study to establish best practice.

\section{Contributors}

Chakravarty initiated the study and participated in review of the literature, data collection, and writing of the paper. Sloan initiated and coordinated the formulation and planning of the study, analysed and interpreted the data and edited the paper. Benchley participated in the protocol design, analysis and interpretation of the data. Chakravarty is the guarantor of the paper.

\section{Authors' affiliations}

D Chakravarty, J Sloan, J Brenchley, Accident and Emergency

Department, The General Infirmary, Leeds, UK

\section{REFERENCES}

1 Leslie IJ, Dickson RA. The fractured carpal scaphoid: natural history factors influencing outcome. J Bone Joint Surg Br 1981;63B:225.

2 Gelberman RH, Wolcock BS, Siegel DB. Current concepts review: fractures and non-unions of the carpal scaphoid. J Bone Joint Surg Am 1989:71A: 1560-5.

3 Duncan DS, Thurston AJ. Clinical fracture of the carpal scaphoid- an illusionary diagnosis. J Bone Joint Surg Br 1985;10B:375-7.

4 Jorgensen TM, Andersen JH, Thommesen $\mathrm{P}$, et al. Scanning and radiology of the carpal scaphoid bone. Acta Orthop Scand 1979;50:663.

5 Belsole RJ, Eikman EA, Muroff LR. Bone scintigraphy in trauma of the hand and wrist. J Trauma 1981,21:163-5.

6 Ganel A, Engel J, Oster Z, et al. Bone scanning in the assessment of the scaphoid. J Hand Surg 1979;4A:540-3.

7 Rolfe EB, Garvie NW, Kahn MA, et al. Isotope bone imaging in suspected scaphoid trauma. Br J Radiol 1981;54:762.

8 Stordahl A, Schjoth A, Woxhott G, et al. Bone scanning of fractures of the scaphoid. J Hand Surg 1984;9B:189-90.

9 Wilson AW, Kurer M J, Peggington JL, et al. Bone scintigraphy in the management of X-ray negative potential scaphoid fractures. Arch Emerg Med 1986;3:235-42.

10 Brismar J. Skeletal scintigraphy of the wrist in suggested scaphoid fractures. Acta Radiol 1988;29:101-7.

11 Young MRA, Lowry JH, Laird JD, et al. ${ }^{99} \mathrm{Tc}^{\mathrm{m}}-\mathrm{MDP}$ bone scanning of injuries of the carpal scaphoid. Injury 1988;19:14-17.

12 Tiel-van Buul MMC, van Beek EJR, Broekhuizen AH, et al. Radiography and scintigraphy of suspected scaphoid fractures-a long-term study in 160 patients. J Bone Joint Surg Br 1993;75B:61-5.

13 Vrettos BC, Adams BK, Knottenbelt JD, et al. Is there a place for radionuclide bone scintigraphy in the management of radiograph negative scaphoid trauma? S Afr Med J 1996;86:540-2

14 Murphy DG, Eisenhaver MA, Powe J, et al. Can a day 4 bone scan accurately determine the presence or absence of scaphoid fracture? Ann Emerg Med 1995;26:434-8.

15 Tiel-van Buul MMC, Roolker W, Verbeeten BWB, et al. Magnetic resonance imaging versus bone scintigraphy in suspected scaphoid fracture. Eur J Nucl Med 1996;23:971-5.

16 Kukla C, Gaebler C, Breitenseger M, et al. Occult fractures of the scaphoid-the diagnostic usefulness and indirect economic repercussions of radiography versus magnetic resonance scanning. $J$ Hand Surg 1997;22B:810-13.

17 Lohman M, Kivisaari A, Vehmas T, et al. MR imaging in suspected acute trauma of wrist bones. Acta Radiol 1999;40:615-18.

18 Hunter JC, Escobedo EM, Wilson AJ, et al. MR imaging of clinically suspected scaphoid fractures. AJR Am J Roentgenol 1997; 168:1287-93.

19 Tiel-van Buul MMC, Broekhuizen TH, van Beek EJR, et al. Choosing a strategy for the diagnostic management of suspected scaphoid fracture. A cost-effectiveness analysis. J Nucl Med 1995;36:45-50. 